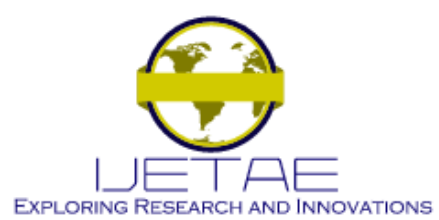

International Journal of Emerging Technology and Advanced Engineering

Website: www.ijetae.com (ISSN 2250-2459, ISO 9001:2008 Certified Journal, Volume 11, Issue 04, April 2021)

Manuscript Received: 05 March 2021, Revised: 12 April 2021, Accepted: 21 April 2021

\title{
Information System for Integrated Medical Records with Access via IOT Technology
}

\author{
Daniel Augusto Pereira ${ }^{1}$, Rodrigo Reina Muñoz ${ }^{2}$ \\ ${ }^{1,2}$ Center for Engineering, Modeling and Applied Social Sciences (CECS), Federal University of ABC - UFABC
}

\begin{abstract}
The objective of this project was the development of an information management system for healthcare purpose using cloud computing and IoT technology. The system was designed to receive data coming from medical instruments, as well as documents issued by healthcare professionals and doctor responsible for the care of a patient. For medical consultation, patients must use an identification card that allows access to the corresponding medical history stored in the cloud. This access can be done through an RFID reader, making it possible to consult patient information on a human-machine interface, where new information can also be registered. For the development of the interface, an application for the Android operating system was created, where information can be accessed, and documents can be stored. Storage was implemented on Google's Firebase ${ }^{\circledR}$ platform. Akyiama's RFID reader integrated in a Raspberry $P i$ was used to access the information. Exemplifying the connection with biomedical instruments, a sen-11574 heart beats sensor was connected to a Raspberry $\mathrm{Pi}$, whose measurements were converted to the I2C format with an ADS-1115 module. Results obtained are very promising to be applied in the automation of hospitals, first aid and private assistance in medical offices. the main benefit for patients is the fact that they can keep their medical history in the cloud, which can prevent loss of patient information in usual cases such as changing doctors, offices, or hospitals, which is commonly the case. Therefore, the system allows the preservation of the patient's historical record. The medical care system would make it more effective as it facilitates the work of medical professionals and assists in the patient's day-to-day life with updated health information.
\end{abstract}

Keywords - E-health, IoT, Medical Records, Firebase Cloud Computing.

\section{INTRODUCTION}

Information systems in the health area are used for the storage, retrieval and use of biomedical data for problem solving and decision making, having a prominent role in modern medicine, making the medical services provided more efficient and ensuring greater patient safety [1].
Although the dissemination of health information systems has grown in the last decade, it is also pointed out in [1] the lack of systems integration, slow adoption of information technology and resistance to the use of new technologies and process redesign, as situations to be improved. Another problem to be solved, according to a paper reported in [2], is the need for a user-friendly interface, for both doctors and patients. Within the context of modern information systems, one of the methods of integrating computer systems with the physical environment is the Internet of Things (IOT), a term coined to describe the connection via the Internet of objects and wireless sensor networks. The interest in devices that work with this concept has grown, as mentioned in [3] pointing out the presence of 25 billion devices connected to the internet in 2021.

Among the areas of main applications of IOT, there is health, where this technology can be used in remote monitoring, creation of profiles and histories, medical records portability, assisted and early diagnoses, activation of emergency units, among other applications [4].

According to the Medical Records Institute in the USA, there are five stages involved in digitizing medical records - automated, computerized, and electronic medical records, electronic patient records and electronic health records. Currently, most hospitals and clinics are in the phase of computerized medical records [5]. According to the World Health Organization (2012), [6], the objective of collecting and storing patient information is to make it available for decision making at a point of care or for management and policy development. It is important to realize that most patients attend more than one point of care, sometimes distinguishing between general and emergency care centers and specialized care. Users of healthcare systems can also move from one location to another, including from country to country. It is essential that individual information is available at all points of care and in all places for analysis. 


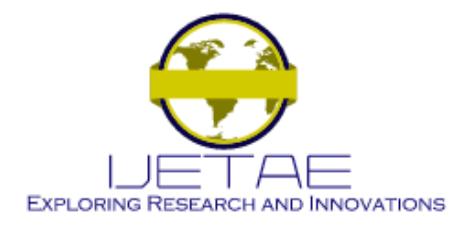

\section{International Journal of Emerging Technology and Advanced Engineering Website: www.ijetae.com (ISSN 2250-2459, ISO 9001:2008 Certified Journal, Volume 11, Issue 04, April 2021)}

Considering the mentioned aspects, the present work aims at the development of a medical information management system, which can receive data from hospital instruments, documents issued by the hospital and doctors, so that the patient has a card that allows access to your medical history stored in the cloud. This access can be done through a RFID reader, making it possible to consult patient information on a human-machine interface, where new information can also be registered.

It is necessary to say that because this project is in its initial stage of development, has no commercial purpose. Connected with this, the next considerations are highlighted:

- Login security: For login purposes, the Firestore Authentication service of Firebase ${ }^{\circledR}$ was used to manage in transit user credentials. This offers the ability to manage users and passwords and enable the login of these accounts securely.

- Patient's information data: Patient's data were stored in Cloud Firestore, Realtime Database and Cloud Storage of Firebase ${ }^{\circledR}$. This ensures that private information will be dealt safely. For these services, Firebase ${ }^{\circledR}$ encrypts the data stored, improving its safety.

- Mobile APP information: Considering that data can be ported in a mobile application, and many countries do not allow to travel their data outside of their country server, it is necessary to emphasize that this aspect was not considered here since the system is in its initial stage of development, aiming to show the technical concept of an integrated information system for medical records. In this way, aspects related to national and international regulations were considered out of the scope of this project.

Next sections describe the paper organization as follows: Section II describes the architecture conceived to the system implementation, and the aspects related to the technology used in the implementation of the system are briefly described. Section III describes the implementation of the hardware and software developed to the system. Also, main results obtained are highlighted in this section. Section IV presents the conclusions of the medical records system designed.

\section{Materials AND Methods}

This section contains a description of how the system works, the hardware used and its connections, the dataflow through the system, software and cloud computing platform used.

\section{A. System flowchart}

The system was designed to integrate the biomedical sensor and RFID reader on the same Raspberry computer, which communicates with the cloud database. The Android application interacts with the cloud and can receive and send information. Figure 1 show the flowchart made to implement the system.

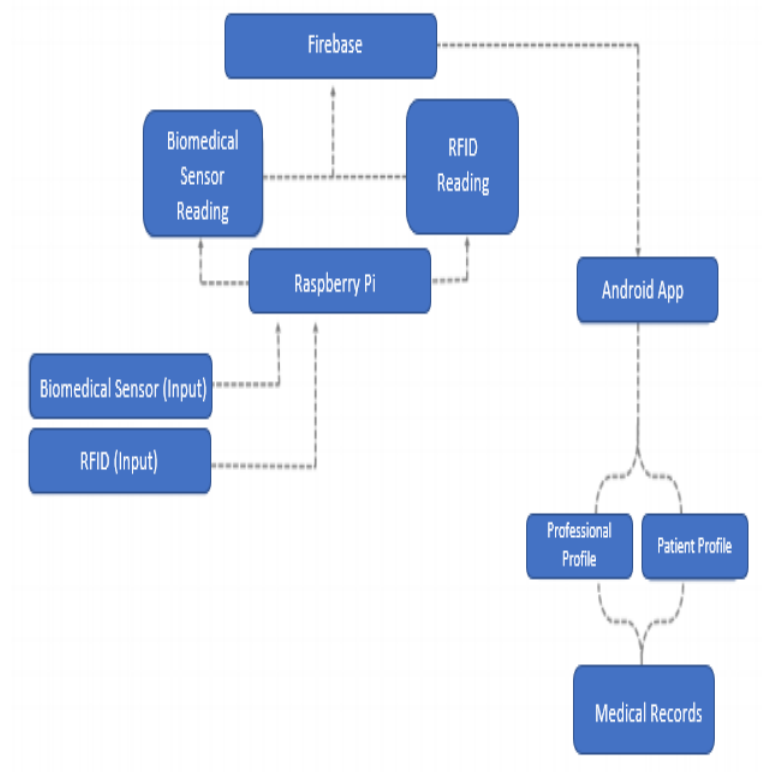

Figure 1. General system flowchart

As shown in figure 1, the dataflow initiates with an electronic hardware interface for acquisition of data from the RFID card of a patient and biomedical sensor used on the healthcare point. These signals are read and transmitted via Internet to the Firebase ${ }^{\circledR}$, where the corresponding variables are filled and stored to be later accessed by the user. The access is then done with an Android APP, logging into the profile with the permissions to record and view the information.

\section{B. Hardware for project implementation}

The interconnection diagram for the proposed system can be seen in the figure 2, which illustrates the different components used in this system. Each part of the system is explained in more detail in the following topics.

The operation of the system is explained as follows: Two parallel system are connected to the Raspberry Pi, the RFID Reader, with the Voltage Converter, and the Heartbeat Sensor with the Analogic-to-Digital Converter. 


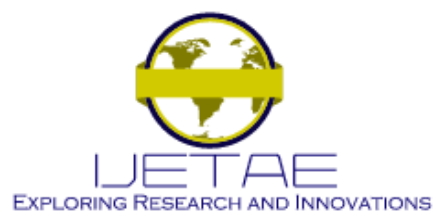

International Journal of Emerging Technology and Advanced Engineering Website: www.ijetae.com (ISSN 2250-2459, ISO 9001:2008 Certified Journal, Volume 11, Issue 04, April 2021)

Once the Python programs are running, they are continuously waiting for a reading of the sensors to be processed.

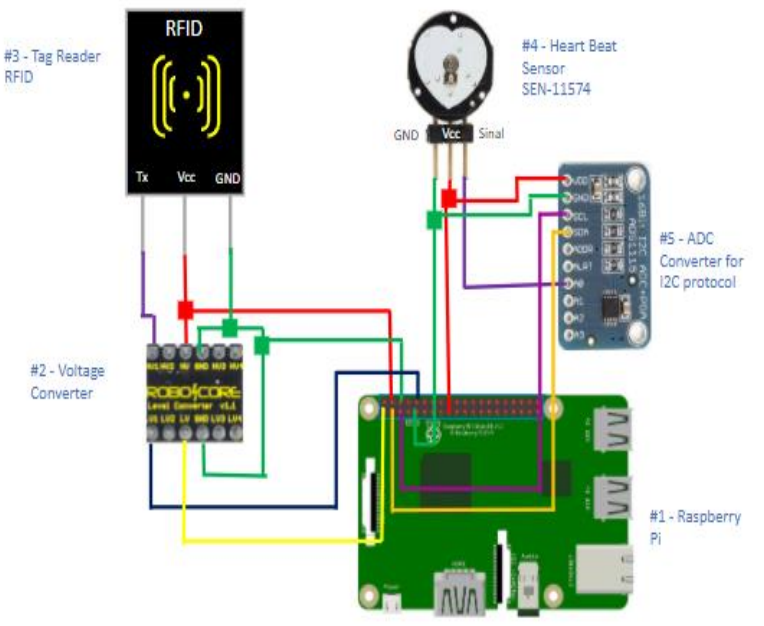

Figure 2. Illustrative diagram of the hardware showing components and interconnections

The RFID reader is triggered when the patient's health card with RFID technology when the RFID card is slid into the reader. The voltage converter is used to receive the $5 \mathrm{~V}$ signal from the RFID reader and convert to the $3,3 \mathrm{~V}$ voltage to be connected to the Raspberry Pi. The heartbeat sensor provides an analogic measurement through photoplethysmography method, which is then converted to a digital protocol by the ADC to communicate with the Raspberry Pi.

\section{C. $R F I D$ reader}

Usually, any physical object that integrates with the Internet of Things (IOT) universe must be uniquely identified. Radio frequency identification (RFID) is one of the most used identification methods in the last decade [7]. An example of an Embedded RFID sensor is the Neoyama AKRFIDCI05-125K-V3, which identifies objects by means of RFID using radio signals, enabling the use of cards to retrieve and store data. The Reader is welded on a plate with 0.1 inches spacing pads, standard for use in protoboards [8].

\section{DC-DC converter}

A logic level converter (DC-DC converter) is an electronic system that works by means of power semiconductors acting as switches associated with passive elements, to control the flow of power from an input source to an output source [9].
The chosen converter performs the voltage conversion by switching a BSS138 MOSFET transistor $\mathrm{N}$ channel connected to the Drain terminal at the highest level $\mathrm{Hv}$ $(5 \mathrm{~V})$ with pull-up resistor $(10 \mathrm{k} \Omega)$ and the Source terminal connected to the lowest level $\mathrm{Lv}(3.3 \mathrm{~V})$ also through resistor $(10 \mathrm{k} \Omega)$.

\section{E. Raspberry Pi}

The Raspberry $\mathrm{Pi}$ is a microcomputer created by the Raspberry Pi Foundation in England [10]. In this project, the model $3 \mathrm{~B}+\mathrm{v} 1.2$ was used, with $1 \mathrm{~GB}$ of RAM and quad-core processor. The Raspberry Pi has a processor that uses the ARM architecture, an acronym for Advanced RISC Machine. These processors in general require fewer transistors when compared to $\mathrm{x} 86$ processors, found in the usual computers. This feature allows the Raspberry to consume less energy and dissipate heat in its processing components more efficiently, allowing for better portability in its design [10].

\section{F. Heart rate sensor}

To measure the pulse of a patient, we have, for example, the photoplethysmography method, performed by the SEN11574 sensor from the manufacturer SparkFun Electronics, which can measure a patient's heartbeat when placed between the index finger or lobe of the ear, as both are surfaces that have a high concentration of circulating blood [11].

\section{G. Analog-to-Digital Converter for I2C protocol}

The analog-to-digital conversion component adopted in this project was the ADS1115, which has 16 bits of resolution and uses the $\mathrm{I} 2 \mathrm{C}$ connection protocol, making its implementation simple and precise. $\mathrm{I} 2 \mathrm{C}$ is a serial protocol for two-wire interfaces, created to connect low-speed interfaces, such as microcontrollers, EEPROMS, digital-toanalog and analog-to-digital converters, input and output interfaces and embedded systems. Initially, this protocol was created by the Philips company and is currently widely used for communication between microprocessed systems [12].

\section{H. Google Firebase ${ }^{\circledR}$}

Google Firebase ${ }^{\circledR}$ is a platform for developing web and mobile applications with high integration to different types of devices. The initial premise of this application is to provide developers with a unified system, containing the tool, infrastructure, database, authentication and security in a single system, supporting the main languages used by the technical community today, such as Java, Javascript and Python. 


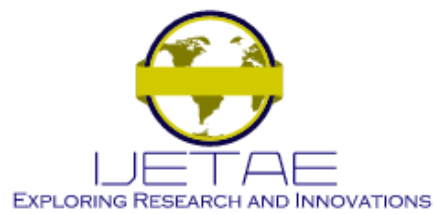

International Journal of Emerging Technology and Advanced Engineering Website: www.ijetae.com (ISSN 2250-2459, ISO 9001:2008 Certified Journal, Volume 11, Issue 04, April 2021)

Firebase provides users with an integrated way to develop and host applications on the Web for free and safely [13].

\section{Android and Android Studio}

The Android platform provides an environment for mobile devices, has its operating system based on Linux and is widely known because of its Open-Source platform, with free access to any developers interested in developing applications aimed at this platform [14]. Android Studio is the main integrated development environment published by Google, with its high integration capacity with any devices using the Android operating system. It has become the main programming environment used to create applications for smartphones, television with access to internet and several other applications currently available [15].

\section{RESULTS AND DISCUSSION}

According to the proposed objective, the electronic information management system (medical records) was developed for the integration of data and biomedical instruments, which is accessible through an Android application that performs the interface between the user and the database. For the identification of patients, RFID cards were used, with the Raspberry Pi being the microcontroller responsible for integrating the information obtained with the database in the cloud. The operation of the developed system can be understood through the block diagram of Figure 3.

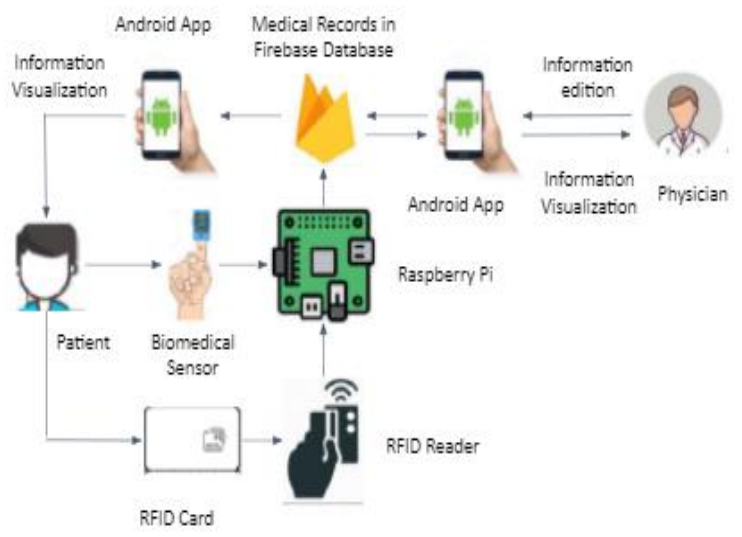

Figure 3: Block diagram of information flow in the System

The patient will be carrying an RFID card for his identification in the system, so that the doctor uses the electronic reader to access the patient's medical records through the application on the smartphone.
The patient can perform an examination on a biomedical sensor connected with the Raspberry, in order to store the reading of this instrument in the cloud. The data shown on the smartphone corresponds to a medical record file accessed from Firebase ${ }^{\circledR}$. Both, doctor, and patient, can access this data, but only the doctor can register and change information.

\section{A. Initial Settings}

Initially, the necessary settings for connecting the RFID reader and the SEN-1174 sensor to the Raspberry Pi were made. The code for establishing serial communication between the RFID reader and the Raspberry was written in Python. Then, the module was added to send the read value to the database in the firebase ${ }^{\circledR}$. Figure 4 shows the hardware of the system implemented.

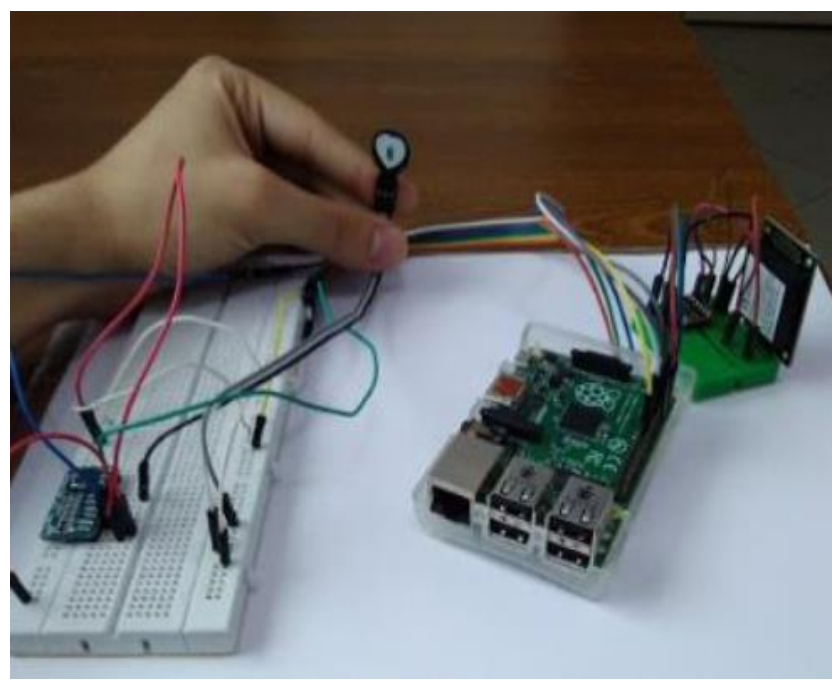

Figure 4 - Prototype of the hardware used for RFID reading and Heart Rate measurement.

For the SEN-11574 sensor, a code developed by Adafruit [16] was used, using the ADS1115 converter for the $\mathrm{I} 2 \mathrm{C}$ protocol. However, the lines needed to send the read value to Firebase ${ }^{\circledR}$ were added to the original code. For this, it is necessary to add a key in JSON format to the Raspberry Pi files, which is generated by the Firebase ${ }^{\circledR}$. The code written is shown in figure 5.

Using the db.reference command it is possible to send a value for the referenced variable in the Firebase database. 


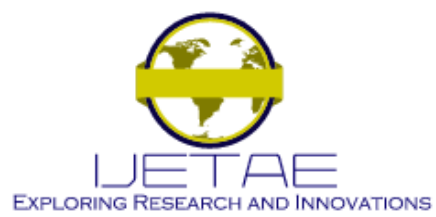

International Journal of Emerging Technology and Advanced Engineering Website: www.ijetae.com (ISSN 2250-2459, ISO 9001:2008 Certified Journal, Volume 11, Issue 04, April 2021)

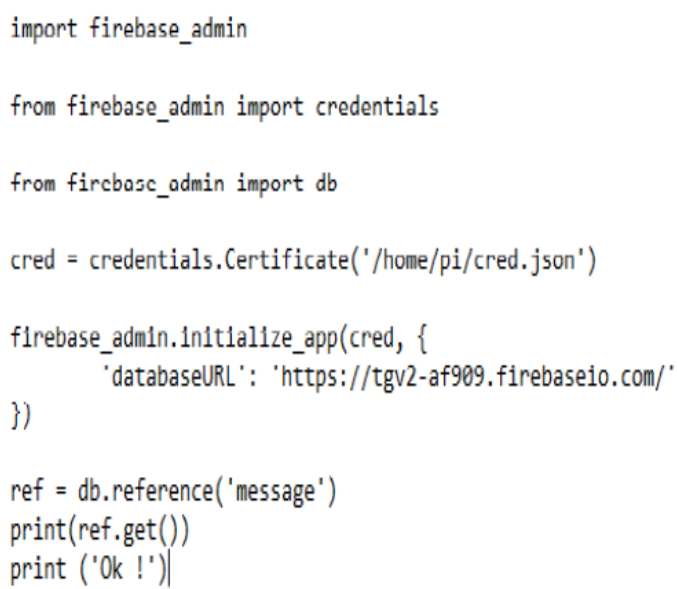

Figure 5. Python code for importing the JSON file into the Raspberry Pi

\section{B. App and Interface screens}

To access the information through the android APP, user interfaces were developed. The links between each developed functionality are shown in the block diagram of figure 6. Following, the interfaces developed are explained in a summarized way, showing examples of the developed APP.

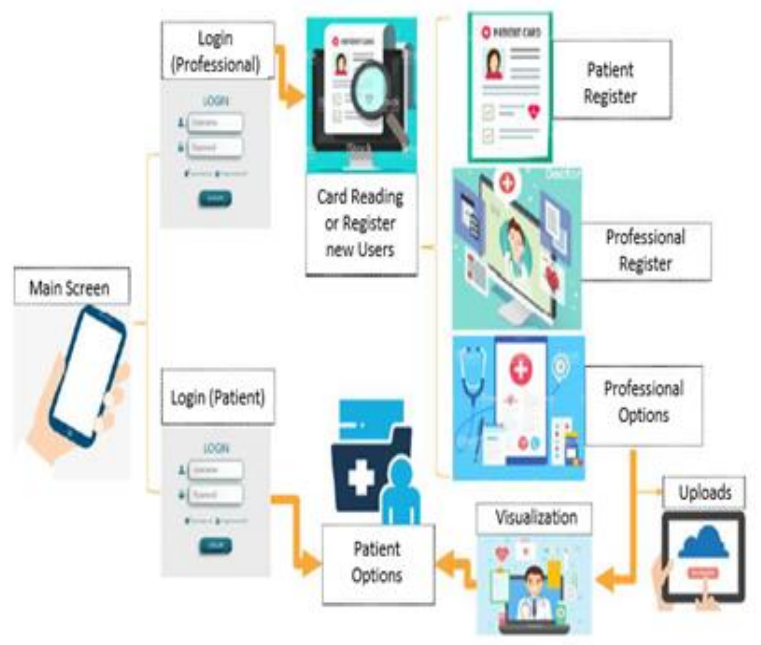

Figure 6. Diagram of the diverse functionalities of the App

\section{Main interface}

In this interface, the user selects the profile with which he wants to access the APP (health professional or patient). Figure 7 shows this part of the APP.

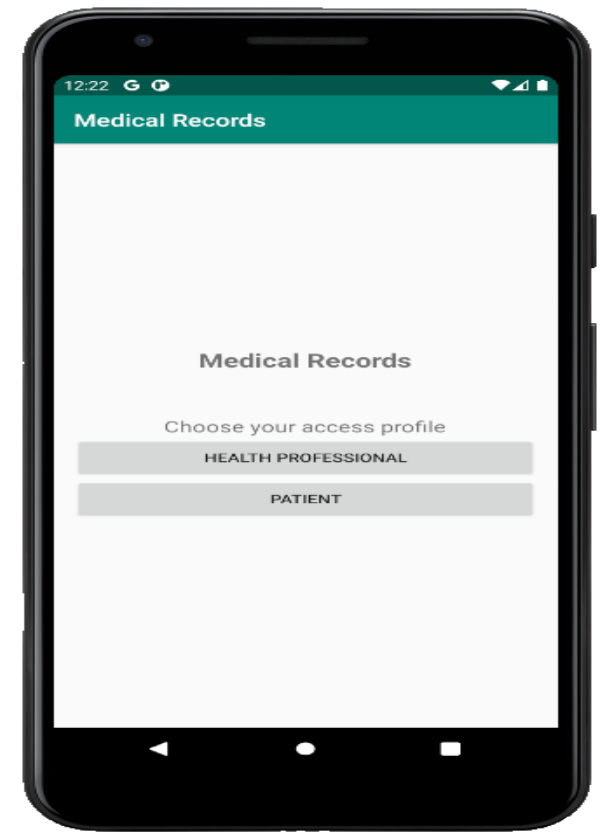

Figure 7. Main interface

\section{Login interface}

This interface has the function of performing user authentication, through e-mail and password, which were previously registered in Firebase ${ }^{\circledR}$ for validation. Figure 8 shows the login interface developed. 


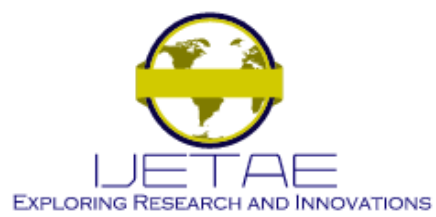

International Journal of Emerging Technology and Advanced Engineering

Website: www.ijetae.com (ISSN 2250-2459, ISO 9001:2008 Certified Journal, Volume 11, Issue 04, April 2021)

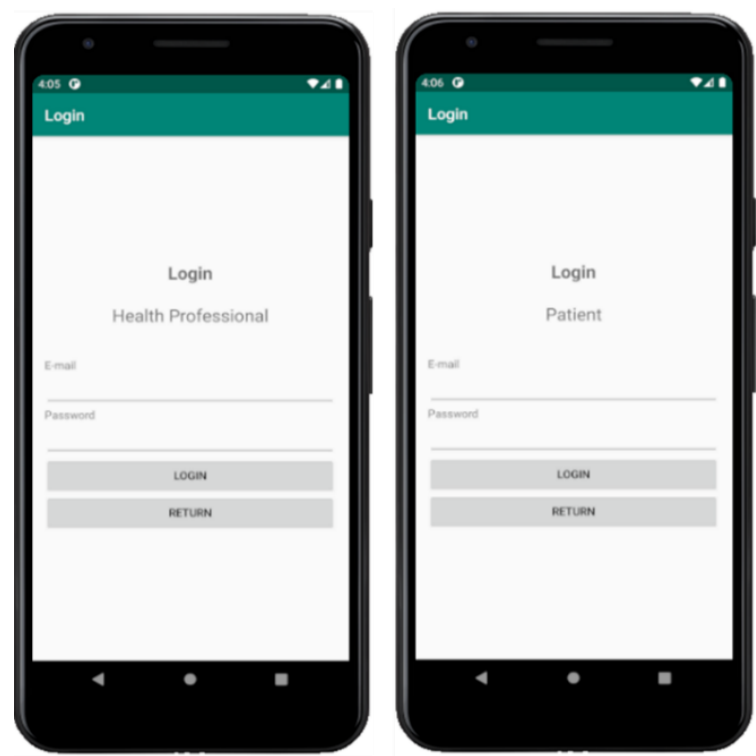

Figure 8. Login interface

Once Login is performed, different interfaces are shown for each user profile. Following are the interfaces developed for the user with a Medical profile.

\section{E. Health Professional interface}

In this interface, the doctor or health professional can choose which services to perform, being able to consult the patient's records, register a new patient or another professional user on the platform. To consult a patient's history, the RFID card must be swiped on the reader, as the APP will be waiting for the receipt of this signal. In the absence of the card, the doctor can enter the patient's identification number. Figure 9 shows the corresponding window that is displayed when the doctor profile is chosen.

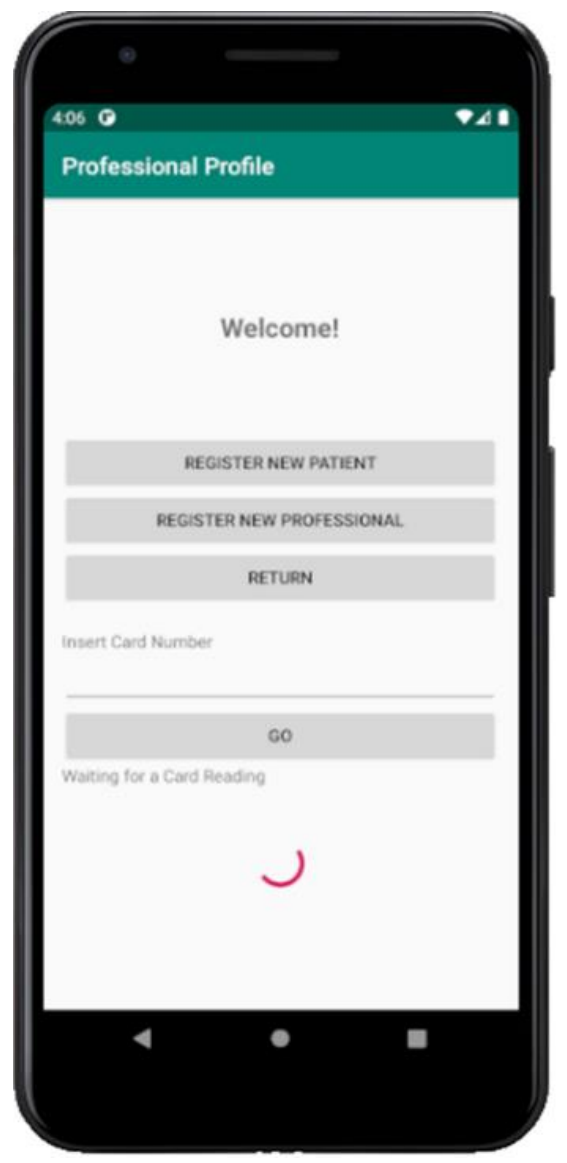

Figure 9 - Health Professional Options Screen

\section{F. Options interface}

After reading or entering the patient's ID, the doctor is directed to a window with options of activities that can be performed. 


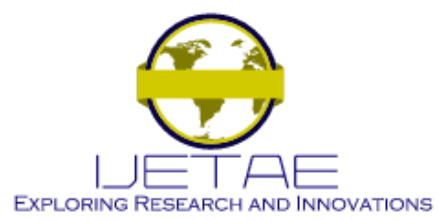

International Journal of Emerging Technology and Advanced Engineering

Website: www.ijetae.com (ISSN 2250-2459, ISO 9001:2008 Certified Journal, Volume 11, Issue 04, April 2021)

For example, view history of values measured by the biomedical sensor, view images stored in the patient's history, view the patient's vaccine history, view saved documents, or perform a new upload of information. Each radio button takes the user of the system to a new window where the saved information is shown. The user with a medical profile can view, add, and delete information if necessary. Figure 10 shows these features.

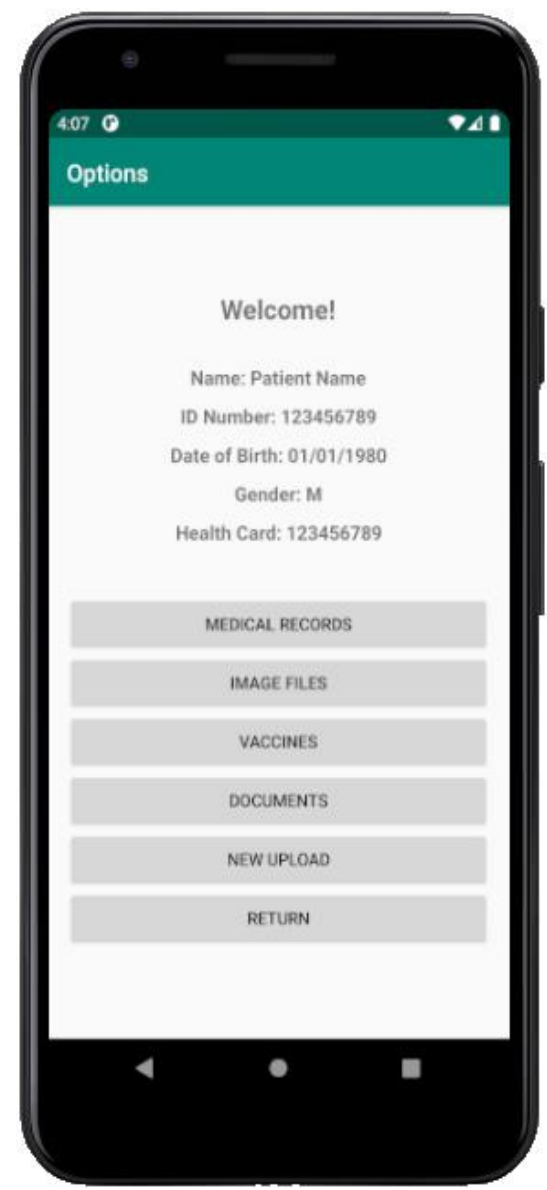

Figure 10. Patient Data and Medical Records Visualization

\section{G. Upload interface}

The upload interface allows the doctor to choose what information he wants to store: a new value measured by a biomedical sensor, a new image, a new document, or a new vaccine information. Each option leads to a new window where the information will be entered and transferred to the database. Figure 11 shows this interface.

To upload a document, a file chooser is opened, allowing the healthcare professional to select a pdf document to save in the firebase ${ }^{\circledR}$ Cloud Storage.
The retrieval of the document is later possible by clicking on the file listed in the visualization window, which redirects the user to the corresponding URL on the browser to download the document. Figure 12 shows examples of choosing a file and downloading it.

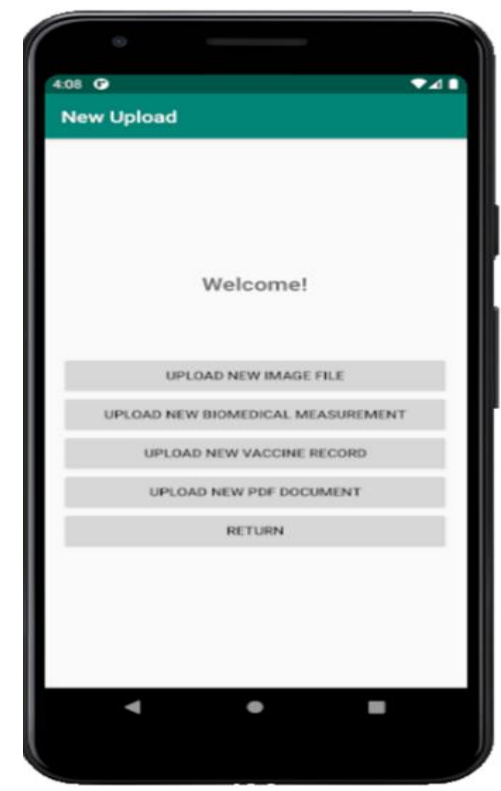

Figure 11. Upload interface

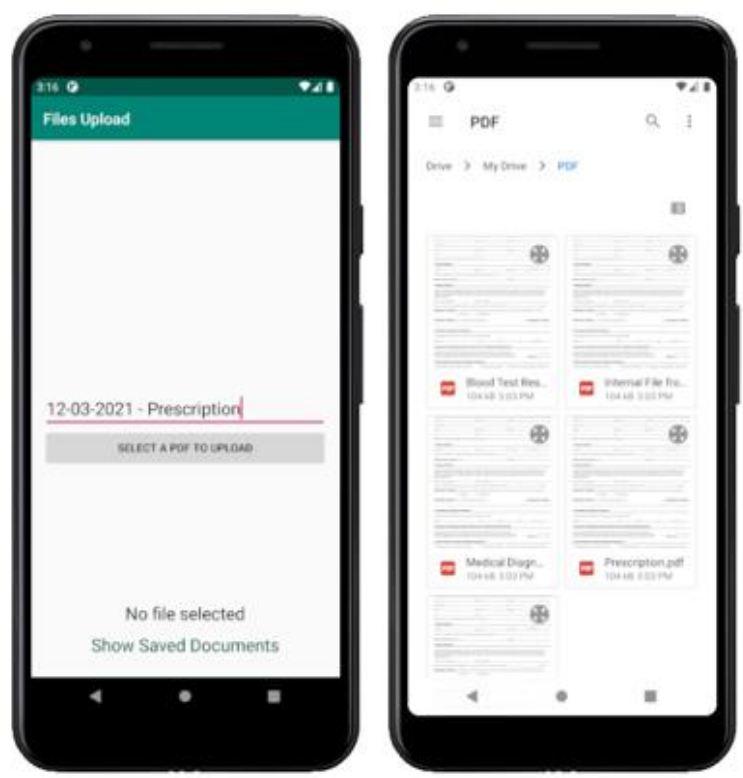

Figure 12. (a) Upload of a document in the cloud 


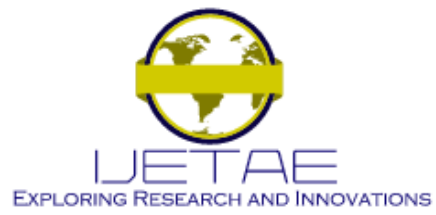

International Journal of Emerging Technology and Advanced Engineering Website: www.ijetae.com (ISSN 2250-2459, ISO 9001:2008 Certified Journal, Volume 11, Issue 04, April 2021)
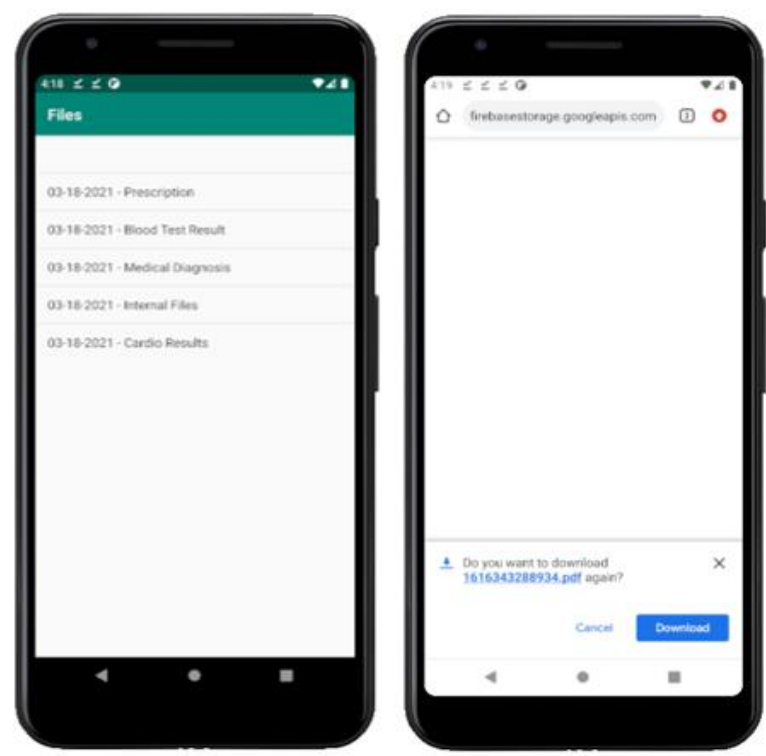

Figure 12. (b) Example of download from Firebase ${ }^{\circledR}$ system

The upload of images is similar. A file chooser is opened, the image is then uploaded to the cloud storage. However, the visualization page is already able to show the image without any download necessary. Figure 13 shows this procedure.

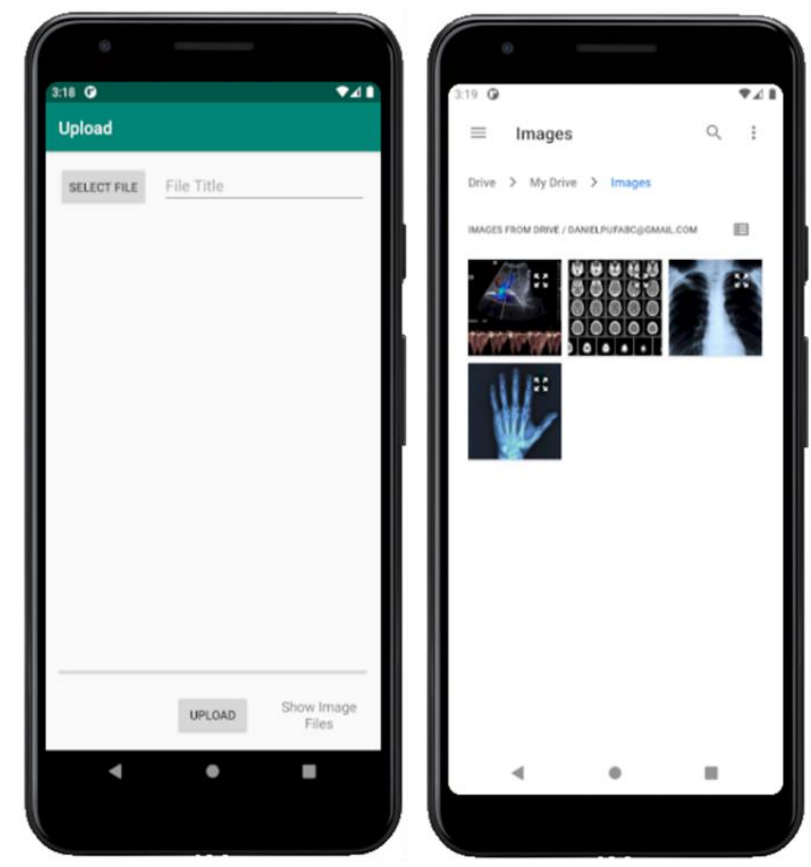

Figure 13 (a). Example of upload of medical images in the Firebase ${ }^{\circledR}$ system (start of upload)
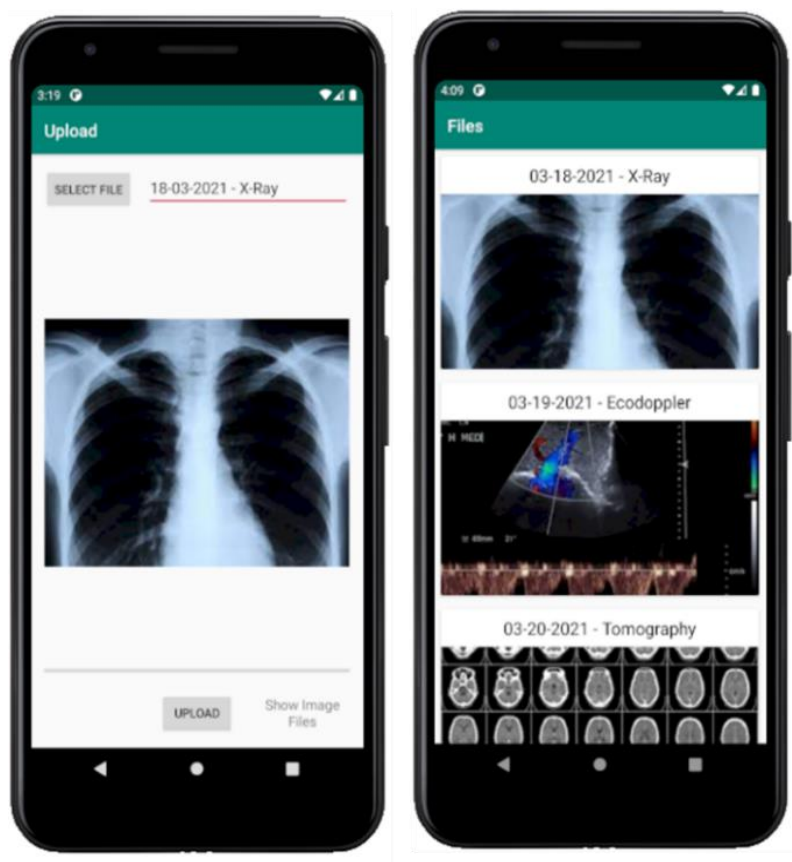

Figure 13 (b). Example of upload of medical images in the Firebase ${ }^{\circledR}$ system (upload completed)

For measurements acquired by a biomedical sensor, the latest value is shown in the upload page. Pressing the button "upload measurement" allows uploading of the measured value if the field for manually input is empty. If a manual measurement was written in the field, then the "upload measurement" button saves the manual input. The vaccines upload is also done by manually filling and pressing the upload button.

For measurements of biomedical vital signs and vaccines history, a ListView is shown with the historical values. Figure 14 shows these interfaces.

\section{H. Registration interface}

In this APP, the health professional is authorized to register new patients who do not yet have a record, or even new professionals to be included into the system. Figure 15 shows this interface.

\section{Patient options interface}

After login, the user is automatically directed to the options page for viewing the history: View measurement history of the biomedical sensor, image history, document history and vaccines. For the patient's profile, there is no option to upload information and the saved information cannot be edited. Additionally, there is a button that takes the patient to the reminder of the interface. Figure 16 shows this part of the APP. 


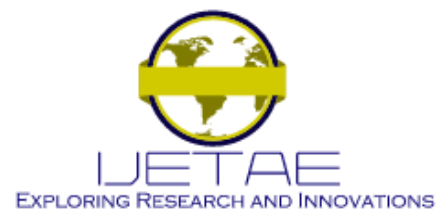

International Journal of Emerging Technology and Advanced Engineering

Website: www.ijetae.com (ISSN 2250-2459, ISO 9001:2008 Certified Journal, Volume 11, Issue 04, April 2021)
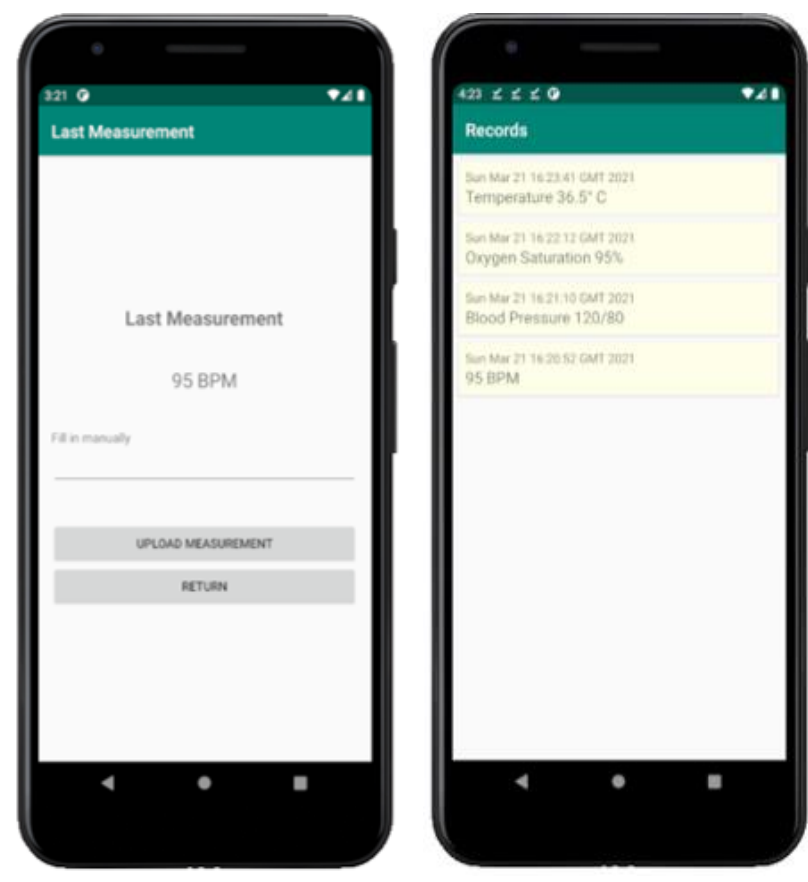

Figure 14. Upload of biomedical measurements

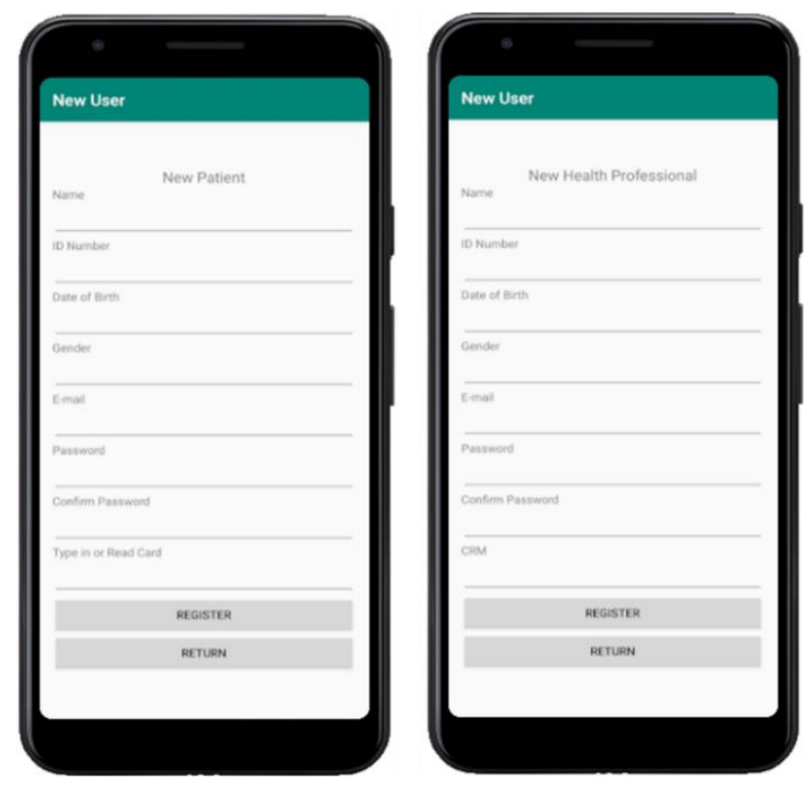

Figure 15. Registration interface

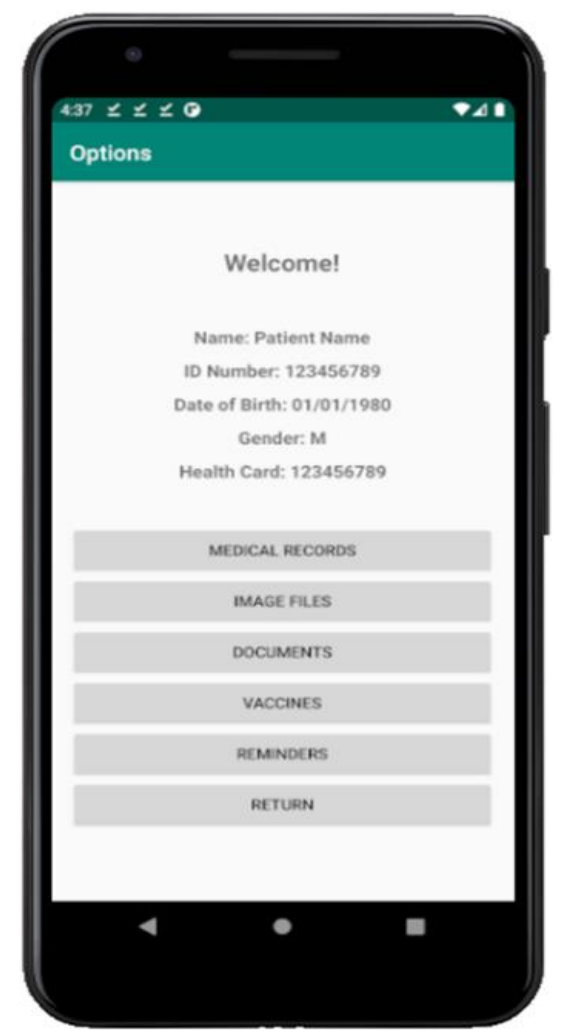

Figure 16. Patient's options

For the Login made with the "Patient" profile, the following interfaces are presented:

\section{J. Reminder interface}

In this interface, the patient can make notes as desired, such as reminders of appointments, medications to be taken, pending vaccinations, etc. Consist of a text box for free filling, which saves the information in "cards". Only the patient has permission to view, upload and delete their reminders. Figure 17 shows this interface.

\section{K. Application - Java Code and settings in Firebase ${ }^{\circledR}$}

The Firebase ${ }^{\circledR}$ system implemented organizes the stored data through "Collections" (groups). Each collection contains saved Documents which have fields for information. It is also possible to insert collections within the Documents, which was used to store data of medical records, vaccines, and patient's saved reminders. 


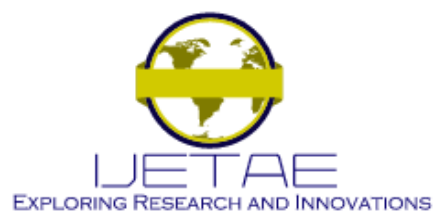

International Journal of Emerging Technology and Advanced Engineering Website: www.ijetae.com (ISSN 2250-2459, ISO 9001:2008 Certified Journal, Volume 11, Issue 04, April 2021)

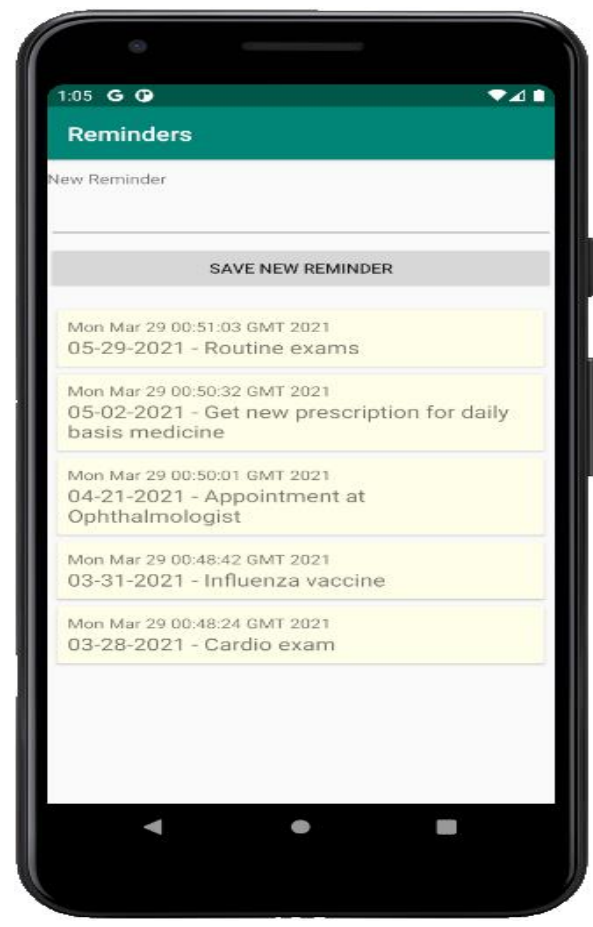

Figure 17. Patient reminders interface

Initially, information regarding patients and doctors was divided into collections. A third collection, named "Profiles", is used to store at registration time, which is the profile related to the registered e-mail. It is used for an initial validation in the Login page if the e-mail attempting to $\log$ have chosen the right profile. Figure 18 shows this interface on Firebase ${ }^{\circledR}$.

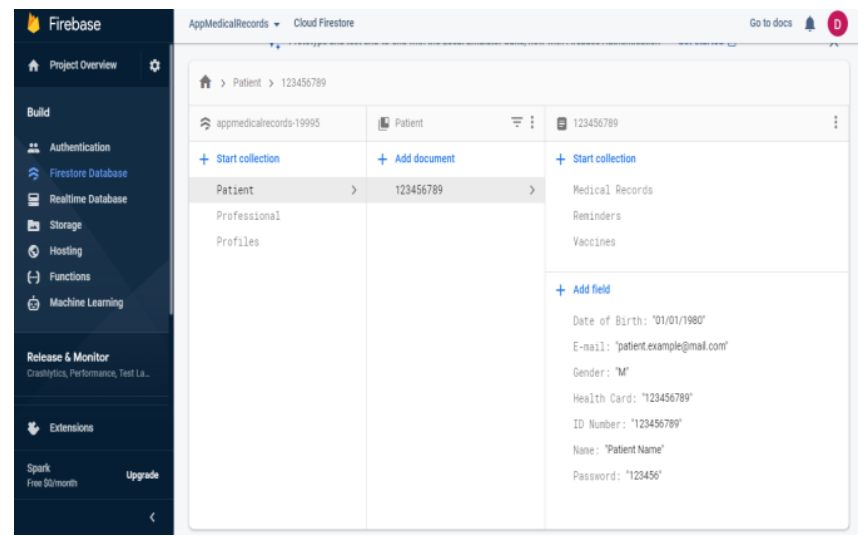

Figure 18. Firebase ${ }^{\circledR}$ Firestore illustration with data from patients and professionals
To fill in this information, the Java Code, implemented in android studio, uses the HashMap class to get the data filled in the app interface and then uses the firebase ${ }^{\circledR}$ package previously imported to send the data to the corresponding collection and document. Figure 19 shows this code.

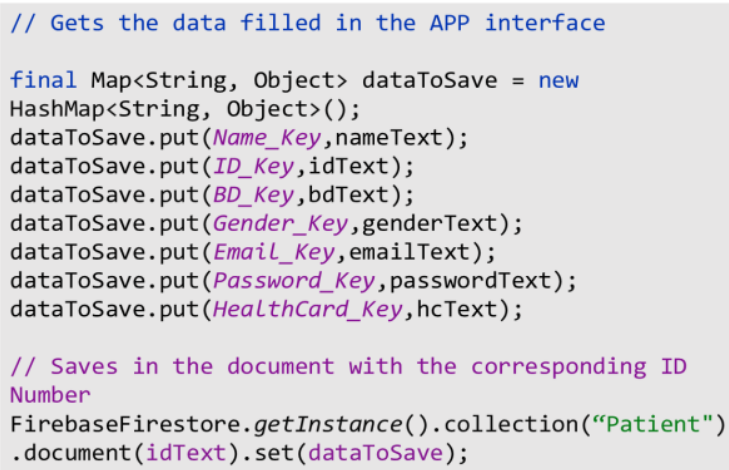

Figure 19. Description of the code of the HashMap function on Java

For the upload of PDF Files and Images, usual methods of opening a file chooser and uploading the files on internet were used. Examples of these codes can be found at Firebase $^{\circledR}$ Guides [17]. The interface on Realtime Database records the title of the file and the corresponding url where the document is saved. When it is requested by the app to download the file, the url is opened in the smartphone web browser and the file is downloaded. Figure 20 shows this interface on firebase ${ }^{\circledR}$.

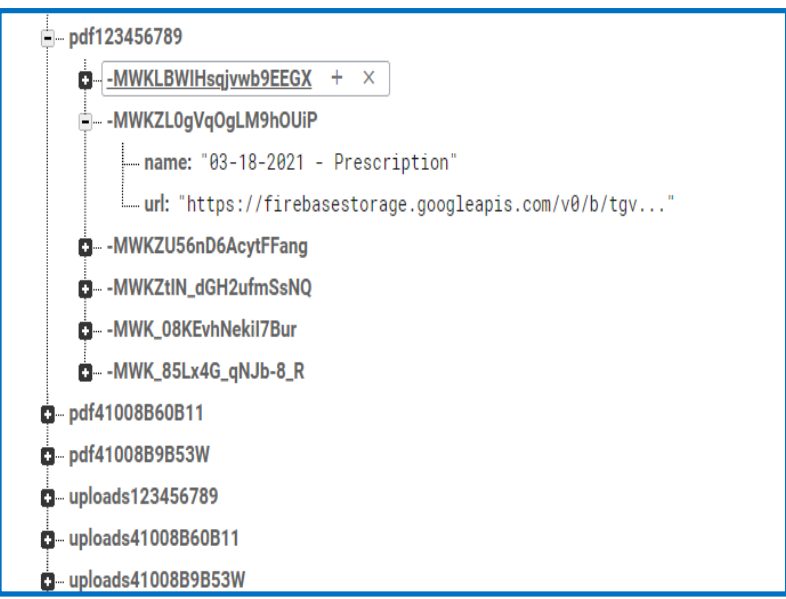

Figure 20. Realtime Database Variables used for documents and image storage of title and URL 


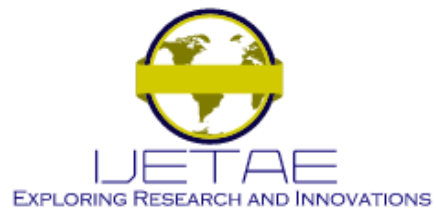

International Journal of Emerging Technology and Advanced Engineering Website: www.ijetae.com (ISSN 2250-2459, ISO 9001:2008 Certified Journal, Volume 11, Issue 04, April 2021)

The files are then recorded on firebase ${ }^{\circledR}$ Cloud Storage named by the health card number of the patient. These files are then downloaded with the URL code saved on Realtime Database. Figure 21 shows the Cloud Storage interface separated by health card number on pdf files.
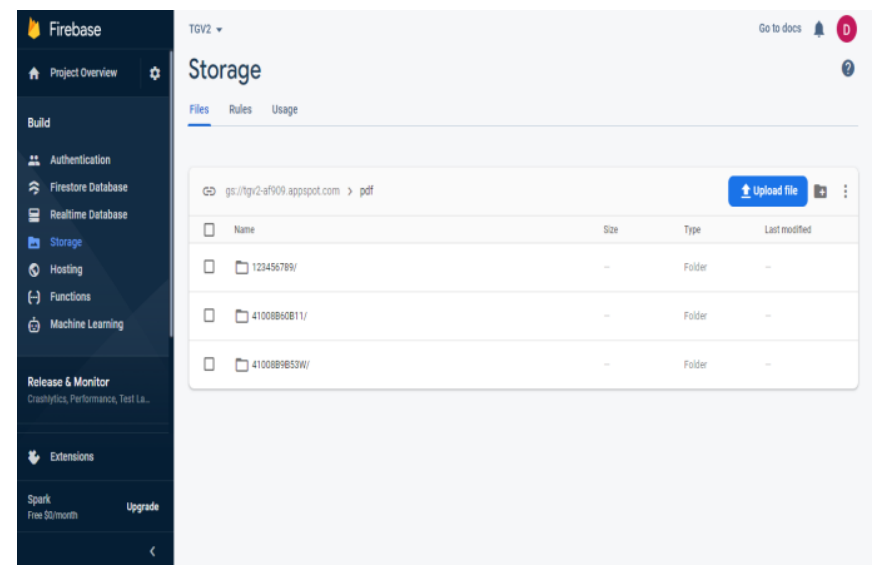

Figure 21. Storage interface

\section{CONCLUSION}

This paper presented the development of a database system for the storage of biomedical information, allowing keeping track of patient's history, thus creating the patient's medical history, a trend within the context of telemedicine. The potential of information technology applied to automate processes in the hospital and health care environment was verified.

The Internet of Things concept was used to integrate physical devices with the information system, creating a physical cyber system where sensors transmit information to the cloud through a microcontroller. For such implementation, RFID technology was used, technology that allows the unique identification of each slave device, in this case the cards and user identification.

As a way of exemplifying the integration of biomedical sensors, it was studied the instrumentation related to the measurement of heartbeat and the method of converting analog measurements to a digital measurement using the I2C protocol.

Additionally, the system designed provided the development and use of a database system in the cloud, which can be accessed in several ways, being compatible with consolidated programming languages, such as Java, Python, Kotlin, to provide an environment that can be used for the development of functionalities that allow the interface with users. It was done the development of an APP for Android operating system.
It is considered, therefore, that the objectives proposed in this initiative were achieved since the system showed proper behavior, allowing to create and maintain medical records of a patient in the cloud, by uploading documents and images, as well as storing in the cloud biomedical measurements, especially those which are easily obtained in an outpatient setting, such as temperature, blood pressure, heart rate, among others.

As contributions in this field, this paper reported a medical record system as an initial approach to the use of cloud computing with the purpose of allow the universal storage of patient's medical records. The introduction of the concept of an independent database, which could be accessed at any health care location and allow people's medical history to be consulted, seeks to instigate the advancement of applications development in this area. The integration of biomedical sensors into the system, showing vital signs directly in the APP and allowing the storage of this data, was exemplified through the heart rate sensor, but other sensors to access vital signs of the patients can be also included in the system. This initial development shows the potential for automation of the medical history storage, as well as the storage of measurements done by biomedical instrumentation in a hospital environment.

\section{Acknowledgments}

Authors thank colleagues Helder Rolim and Alex Oliveira from Federal University of ABC - UFABC, for sharing ideas and experiences during the development of the project. Also thank to Federal University of $\mathrm{ABC}-$ $\mathrm{UFABC}$ for the support given to this work.

\section{REFERENCES}

[1] Perez, Gilberto et al., Determining factors of the adoption of health information systems: a study on electronic medical records (in Portuguese). RAM - Revista de Administração Mackenzie (Online). v. 11, n. 1, p. 174-200, 2010.

[2] Silva, Rogério Oliveira da; OLIVEIRA, José Lucas Sousa de., The internet of things (IOT) with a focus on health (in Portuguese). TECNOLOGIAS EM PROJEÇÃO, v. 8, n. 1, p. 77-85, 2017.

[3] GARTNER. Gartner Identifies Top 10 Strategic IoT Technologies and Trends. 2018. <https://www.gartner.com/en/newsroom/pressreleases/2018-11-07-gartner-identifies-top-10-strategic-iottechnologies-and-trends>. Accessed in 03/21/2021.

[4] Egidio, Lucas, UKEI, Thiago. Internet of Things (IoT): An applicability analysis (in Portuguese). $1^{\circ}$ WSEE-Workshop of embedded systems of ES670. 2015.

[5] Chhatlani, Aakash et al. Portable medical records using internet of things for medical devices. 8th International Conference on Computational Intelligence and Communication Networks (CICN). p. 446-451, IEEE, 2016. 


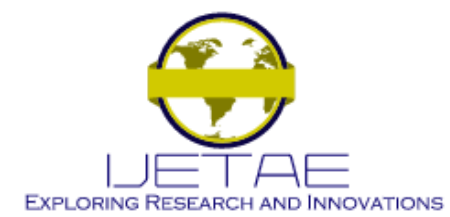

\section{International Journal of Emerging Technology and Advanced Engineering} Website: www.ijetae.com (ISSN 2250-2459, ISO 9001:2008 Certified Journal, Volume 11, Issue 04, April 2021)

[6] World Health Organization. Management of patient information: trends and challenges in Member States: based on the findings of the second global survey on eHealth. Global Observatory for eHealth Series, v. 6, 2012.

[7] Chen, Min, Chen, Shigang. RFID technologies for internet of things. Springer, 2016.

[8] ROBOCORE. Neoyama RFID Reader with Breakout (125kHz) (in Portuguese) Robocore.net, 2019 <https://www.robocore.net/loja/wireless/leitor-rfid-com-breakout>. Accessed at 10/12/2019.

[9] Petry, C.A. Introduction to CC-CC Converters. Federal University of Santa Catarina, Power Eletronics II, 2001.

[10] Donat, Wolfram, Learn Raspberry Pi Programming with Python, ch. 1, p. 1-26, 2018.

[11] Khan, N. et al., Real Time Monitoring of Human Body Vital Signs using Bluetooth and WLAN. IJACSA International Journal of Advanced Computer Science and Applications, v. 7, n. 10, 2016.
[12] I2C INFO - I2C Bus, Interface and Protocol. <https://i2c.info/>. Accessed at 15/10/2019.

[13] Moroney, Laurence. The Definitive Guide to Firebase - Build Android Apps on Google's Mobile Platform, <https://doi.org/10.1007/978-1-4842-2943-9>, v.1, ch. (1-4), p-1-90.

[14] TOP Mobile Operating Systems on Jan 2016. [S.1.], 2016. <https://gs.statcounter.com/\#mobile_os-ww-monthly-201601201601-bar>, acessed at 10/13/2019.

[15] Zapata, Belen Cruz. Android Studio Essentials, v.1, ch. 1 p. 1-13, 2015.

[16] Amaral, Haroldo., Analog to digital converter ADS1115 and LM35 sensor. FilipeFlop, 2018. <https://www.filipeflop.com/blog/conversor-analogico-digitalads1115-sensor-lm35>. Accessed at 10/13/2019.

[17] Firebase Guide. "Upload files to the web" (in Portuguese), https://firebase.google.com/docs/storage/web/upload-files. Accessed at $03 / 21 / 2021$. 